\title{
Raman studies of sol-gel alumina: Finite-size effects in nanocrystalline $\mathrm{AlO}(\mathrm{OH})$
}

\author{
C. J. Doss and R. Zallen \\ Department of Physics, Virginia Tech, Blacksburg, Virginia 24061 \\ (Received 17 August 1992; revised manuscript received 30 August 1993)
}

\begin{abstract}
A systematic Raman-scattering investigation has been carried out on sol-gel alumina prepared by the hot-water hydrolysis and condensation of $\mathrm{Al}\left(\mathrm{OC}_{4} \mathrm{H}_{9}\right)_{3}$, the Yoldas process, as a function of process variables such as the time spent in the sol phase. Nanocrystalline boehmite, $\gamma-\mathrm{AlO}(\mathrm{OH})$, is the principal component of these materials. We have found small but systematic changes, as a function of sol aging time, in the line shape and position of the dominant boehmite Raman band observed in the alumina hydrogels. These spectral changes are interpreted in terms of nanocrystallinity-induced finite-size effects associated with the slow growth of $\mathrm{AlO}(\mathrm{OH})$ nanocrystals in the sol. X-ray-diffraction experiments were used to determine nanocrystal sizes (as small as $3 \mathrm{~nm}$ for gels prepared from fresh sols) and to estimate growth kinetics from the Raman-line-shape results. The Raman peak-position shift is proportional to $L^{-\alpha}$, where $L$ is the average nanocrystal size and $\alpha$ is a Raman-versus-size scaling exponent. For $\mathrm{AlO}(\mathrm{OH})$ we find $\alpha$ to be 1.0 , close to the scaling-exponent values reported for graphite and boron nitride $(\mathrm{BN})$ and different from the values (about 1.5) that describe the reported behavior of Si and GaAs.
\end{abstract}

\section{INTRODUCTION}

A sol-gel route to porous transparent alumina was established by Yoldas in 1975 in a series of widely cited papers. $^{1-4}$ Sol-gel alumina, prepared by the Yoldas process, is the prototypical example of a crystalline (meaning microcrystalline or nanocrystalline) gel. ${ }^{5}$ Figure 1 presents a schematic of the synthesis procedure, as implemented in the present study. Since our study addresses the sol-gel aspects and the structure of the resulting gels, we omit the subsequent thermal treatments used to convert the gels to porous, yet transparent, alumina monoliths. $^{1}$

The Yoldas process for sol-gel alumina includes three main steps: (i) hydrolysis and condensation of an aluminum alkoxide in excess water to form an $\mathrm{AlO}(\mathrm{OH})$ precipitate; (ii) resuspension and dissolution of the precipitate by acid peptization to form a clear, colloidal sol; (iii) concentration of the sol by solvent boiloff, resulting in the formation of a gel.

The initial hydrolysis step is done with hot water, and the peptized sol is kept hot in order to form $\mathrm{AlO}(\mathrm{OH})$ sols and gels rather than precipitates of $\mathrm{Al}(\mathrm{OH})_{3}$ phases. $^{4,6}$ Alumina gels are mainly water and should properly be referred to as alumina hydrogels. Throughout this paper, whenever the term gel appears without a modifier, alumina hydrogel is meant. Dried gels will be specifically referred to as xerogels.

In this paper, we report the results of a systematic Raman-scattering investigation of sol-gel alumina materials as a function of process parameters, particularly the sol aging time ( $t_{s}$ in Fig. 1). Nanocrystalline boehmite $\gamma$-AlO $(\mathrm{OH})$, is shown (by Raman and $\mathrm{x}$-ray measurements) to be the principal nonaqueous component and we have found small but systematic changes, as a function of $t_{s}$, in the line shape and position of the dominant boehmite Raman band. These spectral changes have been in- terpreted in terms of nanocrystallinity-induced finite-size effects associated with the slow growth of $\mathrm{AlO}(\mathrm{OH})$ nanocrystals in the sol phase, and we have used them to determine estimates of characteristic nanocrystal sizes and growth kinetics in this system. This interpretation is supported by $\mathrm{x}$-ray-diffraction experiments (which provide the nanocrystal-size calibration for the Raman changes) and is consistent with crystal-growth models.

Section II describes the experimental techniques used in the synthesis of, and measurements on, the sol-gel alumina materials. The Raman spectrum of crystalline boehmite is described in Sec. III, as a necessary prelude to the measurements on the gels. Raman scattering from alumina gels, and the observation of systematic changes accompanying sol-gel processing, are described in Sec. IV. The interpretation in terms of nanocrystallinity and

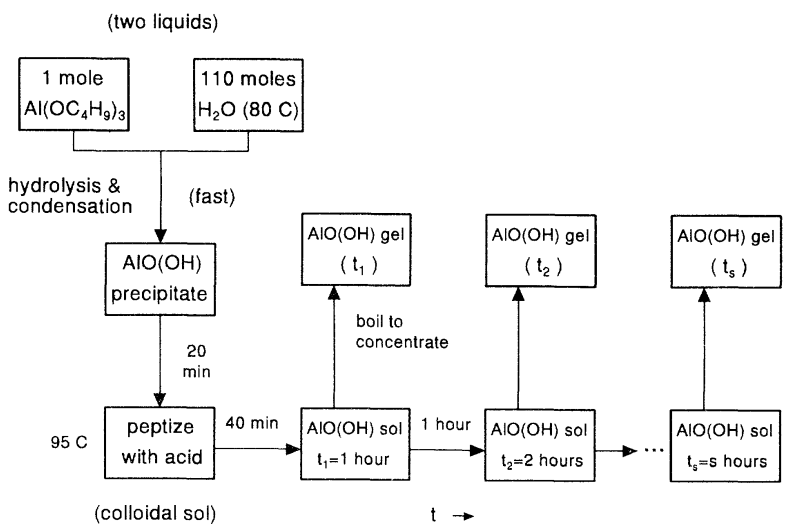

FIG. 1. Schematic of our implementation of the Yoldas process for synthesizing sol-gel alumina. After time $t_{s}$ in the sol phase at $95^{\circ} \mathrm{C}$, gelation was brought on by boiloff-induced concentration. Raman studies were done on hydrogels prepared using various sol aging times $\left(t_{s}\right)$, and the effect of varying the acid peptization step in the synthesis was also studied. 
finite-size effects is given in Sec. V. X-ray-diffraction determination of nanocrystal sizes is described in Sec. VI, and results for the kinetics of nanocrystal growth are given in Sec. VII. Section VIII is a summary of our principal findings.

\section{EXPERIMENT}

\section{A. Preparation of alumina gels}

The Yoldas process for making clear alumina gels, as indicated in Fig. 1, consists of the three main steps listed in the Introduction. The alkoxide we used, aluminum secondary butoxide (ASB), $\mathrm{Al}\left(\mathrm{OC}_{4} \mathrm{H}_{9}\right)_{3}$, was doubly distilled and stored in an inert atmosphere chamber. ASB is a clear, viscous liquid at room temperature. It was heated to reduce its viscosity, drawn into a hypodermic syringe, and added by syringe to the open reaction vessel containing water at $80^{\circ} \mathrm{C}$ under vigorous stirring conditions. The $\mathrm{H}_{2} \mathrm{O}$ :ASB molar ratio was 110 . ASB addition induces immediate hydrolysis and condensation reactions in the water-rich mixture ${ }^{3,4,7}$ and yields an $\mathrm{AlO}(\mathrm{OH})$ precipitate.

The initial hydrolysis temperature of $80^{\circ} \mathrm{C}$ was chosen because it is the highest temperature compatible with avoiding violent boiling caused by the exothermic hydrolysis and condensation reactions. Following the ASB addition, the temperature controller was set to $95^{\circ} \mathrm{C}$. It took about $20 \mathrm{~min}$ for the liquid to reach $95^{\circ} \mathrm{C}$, partly because of the boiloff of the alcohol (secondary butanol) formed as a by-product of the hydrolysis reaction. Twenty minutes after hydrolysis and condensation, acid was added to cause peptization and the dispersion of the precipitate to form a clear, stable, colloidal sol. Two different acids $\left(0.1 \mathrm{~N}_{\mathrm{HNO}_{3}}\right.$ and $\left.0.1 \mathrm{~N} \mathrm{HCl}\right)$ and two different acid concentrations $(R=0.07$ and $R=0.14$, where $R$ is the acid:ASB mole ratio) were used in variations of the peptization procedure.

Following acid addition, the reaction vessel was closed (except for a very small syringe needle hole to equalize pressure) to prevent solvent evaporation. The sol was then maintained, with continual stirring, at $95^{\circ} \mathrm{C}$. At selected times, a sol sample was removed by pipette from the reaction vessel and boiled (for about $10 \mathrm{~min}$ ) in a small open beaker to remove water and concentrate. With sufficient water removal, the sol becomes viscous and approaches the gel point. The viscous sol was then transferred to a glass vial, sealed to retain the remaining water, and allowed to cool to room temperature. It then gelled within a few minutes.

These hydrogels are about $90 \%$ water, by weight. ${ }^{3}$ Early gels (gels made from sols aged for only a few hours) are slightly cloudy; later gels (made from sols aged for many hours) are clear. The cloudiness is attributed to incomplete peptization for short sol aging times. Once a gel is formed, its degree of cloudiness or clarity does not change, nor does its Raman spectrum. As described in Sec. IV, Raman studies reveal structural differences in the gels, which depend on the amount of time spent by the parent sol in the reaction vessel.

\section{B. Preparation of related materials}

A series of samples were prepared with a procedure similar to that of Fig. 1, with the key difference of omitting the acid addition peptization step. These materials remain as precipitates, in contact with the hot, stirred, aqueous bath in the form of a cloudy suspension. At selected times, a sample of the suspension was removed and centrifuged to remove most (about 75\%) of the water. The resulting white mush was then oven dried at 110 ${ }^{\circ} \mathrm{C}$ for $24 \mathrm{~h}$, yielding brittle, white chunks. Raman studies of these dried precipitates (Sec. IV) reveal that their structure, like that of the gels, also depends on history, the time spent in the reaction bath.

In addition to the hydrogels and the precipitates, a few other samples were investigated. Portions of some of the hydrogels were subjected to a $110^{\circ} \mathrm{C}$ oven drying step (like that used for the precipitates), removing their water content and yielding xerogels in the form of transparent chunks. An unpeptized precipitate (referred to as a $130^{\circ} \mathrm{C}$ precipitate) powder sample was made by adding ASB to $\mathrm{H}_{2} \mathrm{O}$ (ASB: $\mathrm{H}_{2} \mathrm{O}$ molar ratio was 110 ) and processing the sol in a pressure vessel at $130^{\circ} \mathrm{C}$ for about five days. The sol was then dried in an oven at $110^{\circ} \mathrm{C}$. The product of this process was a white powder. A sample of crystalline boehmite powder (composed of micron-size crystals), prepared by autoclaving aluminum metal in excess water, was provided by Alcoa laboratories. X-ray diffraction confirmed that the material was wellcrystallized boehmite.

\section{Raman-scattering experiments}

Raman spectra (Stokes spectra) were obtained at room temperature, using a SPEX 1403 Raman spectrometer with a GaAs photocathode photomultiplier and photoncounting electronics. A cw argon-ion laser, operating at 488.0 or $514.5 \mathrm{~nm}$, was the excitation source. The laser power at the sample was approximately $80 \mathrm{~mW}$. We used an instrumental bandwidth of $3.8 \mathrm{~cm}^{-1}$ and a $0.5 \mathrm{~cm}^{-1}$ grating step size. Spectra were obtained in the $90^{\circ}$ scattering configuration, collecting both polarizations of scattered light. For the hydrogels, spectra were taken with the gel held in its vial, laser light entering from the bottom and scattered light collected through the side (each sample was scanned for a total of $40 \mathrm{~min}$ ). The beam focus was inside the gel; no spectral features attributable to the glass vial were observed. Xerogels and precipitates were measured in air, glued onto a capillary tube. The crystalline boehmite powder and the $130^{\circ} \mathrm{C}$ precipitate powder were held in capillary tubes for $\mathrm{Ra}-$ man measurements.

The experimental Raman linewidth is a convolution of the actual linewidth $\Gamma$ and the instrumental bandwidth $\Gamma_{\text {inst }}$, via $\Gamma_{\text {expt }}=\left(\Gamma^{2}+\Gamma_{\text {inst }}^{2}\right)^{1 / 2}$. In the analysis presented, ${ }^{8}$ we use the (deconvoluted) results for the estimated actual linewidth $\Gamma$.

\section{X-ray-diffraction experiments}

$\mathrm{X}$-ray-diffraction traces were obtained at room temperature, using a Scintag XDS-2000 powder diffractometer 
and $\mathrm{Cu} K \alpha$ radiation with a wavelength of $1.54 \AA$. The $\mathrm{x}$-ray measurements were carried out on powder samples (prepared by gentle grinding of the dried precipitate or hydrogel) placed on an oriented quartz substrate. Diffraction done directly on hydrogel films cast on the substrate missed several boehmite peaks because of drying-induced preferred orientation, ${ }^{9}$ so powder samples were prepared by room-temperature drying of the hydrogels, followed by gentle grinding.

Crystallite sizes were determined by the Scherrer equation, ${ }^{10}$

$$
B(2 \theta)=\frac{0.94 \lambda}{L \cos \theta},
$$

where $L$ is the microcrystallite size, $\theta$ is the peak position, and $B(2 \theta)$ is the linewidth. Linewidth corrections for x-ray instrumental broadening were performed in the same manner as they were for the Raman linewidths. ${ }^{10}$

\section{RAMAN SPECTRUM OF BOEHMITE [AIO(OH)]}

Boehmite, $\gamma-\mathrm{AlO}(\mathrm{OH})$, is the predominant phase present in the alumina gels formed by the Yoldas process of Fig. 1. It is, therefore, necessary to consider first the Raman spectrum of this crystal form. The crystal structure of boehmite is shown in Fig. 2. ${ }^{11,12}$ The structure is orthorhombic, space group $C m c m\left(D_{2 h}^{17}\right)$, with two $\mathrm{AlO}(\mathrm{OH})$ formula units per primitive cell. The symmetric unit cell shown in Fig. 2 contains two primitive cells. The structure is based on extended layers that run perpendicular to the $b$ axis; parts of two layers are shown in Fig. 2. There is ionic and covalent bonding within each layer, but only hydrogen bonding between layers. Within each multilevel, strong-bonding, layer there are two planes of aluminum atoms and four planes of oxygen atoms. Each aluminum is bonded to six oxygens in a distorted octahedron. The interior oxygens (those on the two inner oxygen planes) are each bonded to four aluminums in a highly distorted tetrahedron. The outer oxygens (actually hydroxyls, since each carries a hydrogen) are each bonded to only two aluminums.

An appropriate reference for comparison is $\alpha-\mathrm{Al}_{2} \mathrm{O}_{3}$ (corundum, also called sapphire or alumina). In the three dimensional (3D)-network structure of $\alpha-\mathrm{Al}_{2} \mathrm{O}_{3}$, each aluminum is coordinated to six oxygens and each oxygen to four aluminums. The average coordination is 4.8 , compared to an average Al-O coordination of 4.0 within a boehmite layer. For $\alpha$-alumina, $\operatorname{Raman}^{13}$ and infrared $^{14}$ studies place the optical phonons in the range from 380 to $750 \mathrm{~cm}^{-1}$. For boehmite, the lower coordination is expected to extend the Al-O optical-phonon regime to lower frequencies, and we find this to be the case.

The strongest Raman line we observe in $\gamma$-AlO $(\mathrm{OH})$ occurs at $363 \mathrm{~cm}^{-1}$. Its strength, as well as the observation (discussed below) that its depolarization ratio is zero, marks it as a fully symmetric mode ( $A_{g}$; there are three of this symmetry), in which all aluminums and oxygens move parallel to the $b$ axis. The six vertical arrows in Fig. 2 show one possible $A_{g}$-symmetry vibrational eigenvector (we can treat each hydroxyl as a rigid entity, for a low-frequency mode, so only six arrows are needed) which has intralayer-shear bond-bending character, but a definitive assignment awaits a more detailed force-field analysis.

Figure 3 presents Raman-scattering results obtained from boehmite powder composed of micron-scale crystallites. ${ }^{15}$ At these relatively large particle sizes, the observed spectrum should be indistinguishable from that of the bulk crystal. This is supported by the sharpness of the Raman lines in Fig. 3. (An x-ray-diffraction spectrum for this sample, displaying sharp lines, is shown in Sec. VI.)

The spectral region shown in Fig. 3 contains the lattice-mode regime, i.e., the modes are predominantly Al-O vibrations. Table I lists the peak positions, linewidths, and relative intensities of the Raman lines of boehmite. The linewidth given is the full width at half maximum (FWHM), after correcting for the instrumental broadening. The sharp, intense line at $363 \mathrm{~cm}^{-1}$ is the dominant spectral signature of boehmite. The line-shape

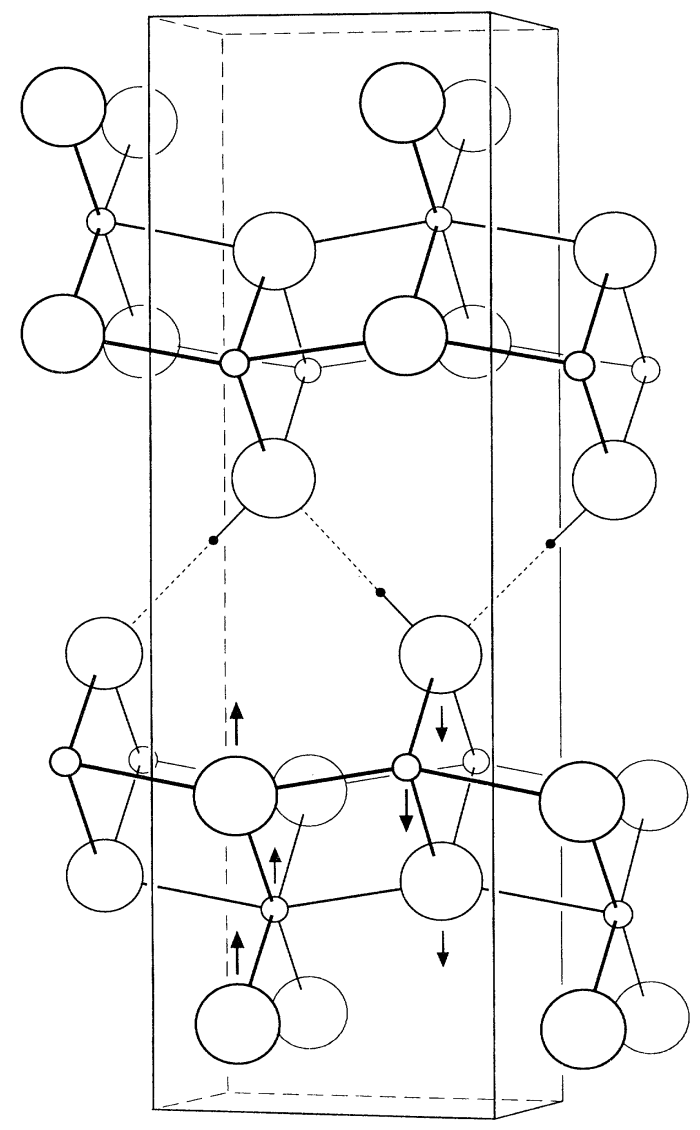

FIG. 2. The crystal structure of boehmite $\gamma-\mathrm{AlO}(\mathrm{OH})$. This is a perspective view from a distance of $50 \AA$, looking along a direction $15^{\circ}$ off the $a$ axis in the ac plane (see Ref. 12). The cell shown is a symmetric unit cell, which contains two primitive cells. Oxygens are represented by the large spheres, aluminums by the small spheres, and hydrogens by the solid dots. The six arrows, attached to the aluminums and oxygens within one primitive cell, represent a vibrational eigenvector having the full crystal symmetry. 


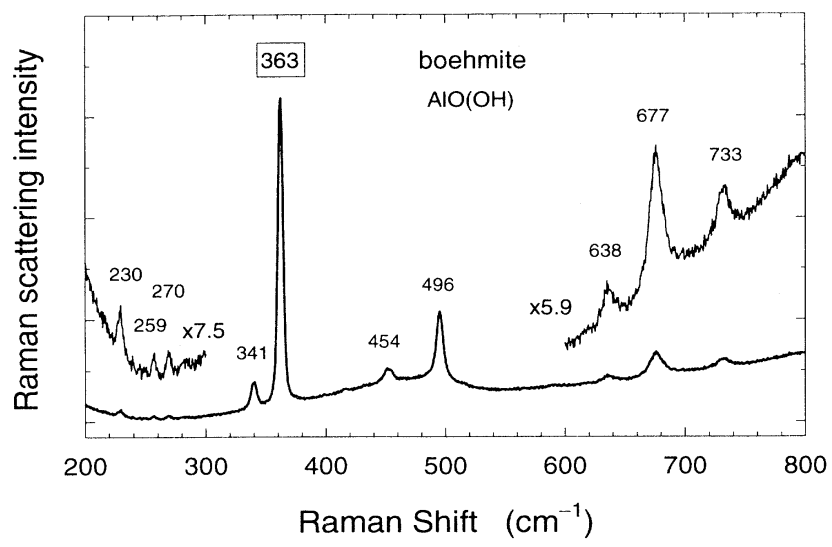

FIG. 3. The Raman spectrum of crystalline boehmite in the Al-O regime. Scattered light intensity is shown plotted against photon-energy downshift, in wave-number units, relative to the laser line (the $488.0 \mathrm{~nm}$ line, for this case).

variations of this feature, as observed for the gel samples, will play an important role in our analysis.

Figure 4 shows the polarization dependence of the 363 $\mathrm{cm}^{-1}$ line. (For this nanocrystalline sample, the line is downshifted to $359 \mathrm{~cm}^{-1}$.) These spectra are for a hydrogel, peptized with $\mathrm{HNO}_{3}(R=0.07)$, and aged in the sol phase for $48 \mathrm{~h}$. These clear hydrogels are convenient for depolarization studies because of their low scattering background and the random orientation of the crystallites. The clean diagonal polarization exhibited in Fig. 4 supports the fully symmetric $A_{g}$-symmetry interpretation for the $363 \mathrm{~cm}^{-1}$ Raman band.

In comparing our results to earlier reports on boehmite $^{16-18}$, we have discovered that considerable confusion has existed in terms of Raman lines attributed to boehmite and boehmite gels. Specifically, we have found that several high-frequency lines, which have masqueraded in the literature as boehmite modes, are actually internal modes of the $\mathrm{NO}_{3}{ }^{-}$nitrate ion. Figure 5 compares

TABLE I. Raman peak positions for crystalline boehmite, $\mathrm{AlO}(\mathrm{OH})$.

\begin{tabular}{ccc}
\hline \hline $\begin{array}{c}\text { Frequency } \\
\left(\mathrm{cm}^{-1}\right)\end{array}$ & $\begin{array}{c}\text { Linewidth }^{\mathrm{a}} \\
\left(\mathrm{cm}^{-1}\right)\end{array}$ & $\begin{array}{c}\text { Relative } \\
\text { intensity }\end{array}$ \\
\hline 230 & 3.2 & Weak \\
259 & 2.6 & Weak \\
270 & 2.4 & Weak \\
341 & 3.2 & Medium \\
363.3 & 3.0 & Very strong \\
454 & 7.3 & Medium \\
496 & 6.3 & Strong \\
638 & 10.9 & Weak \\
677 & 12.4 & Medium \\
733 & 9.5 & Weak \\
3079 & 47 & Medium \\
3219 & 37 & Medium \\
\hline \hline
\end{tabular}

aThe linewidth means full width at half maximum (FWHM), and is corrected for the instrumental broadening via $(\mathrm{FWHM})_{\mathrm{obs}}^{2}=(\mathrm{FWHM})_{\mathrm{actual}}^{2}+(\text { instrumental spectral slit width })^{2}$.

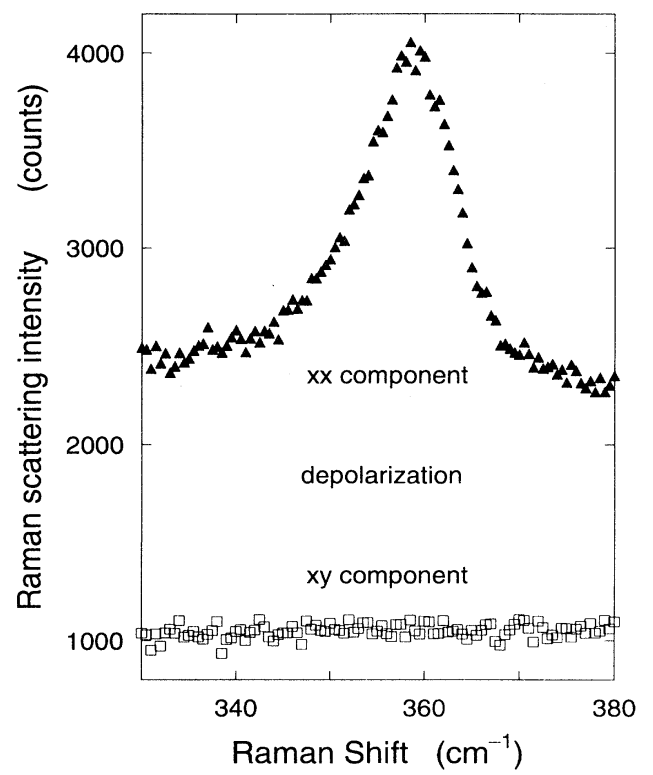

FIG. 4. The polarization dependence of the $363 \mathrm{~cm}^{-1}$ line for boehmite. (For this nanocrystalline sample, the line is downshifted to $359 \mathrm{~cm}^{-1}$.) These spectra (obtained with $514.5 \mathrm{~nm}$ excitation) are for a hydrogel, peptized with $\mathrm{HNO}_{3}(R=0.07)$ and aged in the sol phase for $48 \mathrm{~h}$. This clean depolarization behavior supports the fully symmetric $A_{g}$ assignment for this band.

spectra that we have observed for boehmite crystals (lower panel), an alumina hydrogel or "boehmite gel" (middle panel), and nitric acid (upper panel). The gel was prepared as in Fig. 1, using $\mathrm{HNO}_{3}$ as the peptizing acid. Note the four Raman bands indicated by the vertical

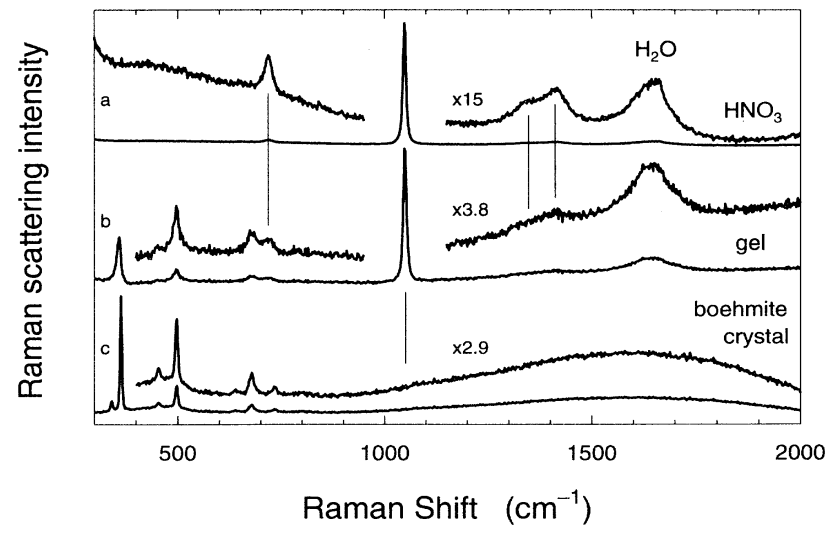

FIG. 5. Comparison of the Raman spectra of (a) nitric acid, (b) an alumina hydrogel, and (c) crystalline boehmite. (These spectra, as well as those shown in the following figures, were obtained with $514.5 \mathrm{~nm}$ excitation.) The spectrum shown in (a) was obtained for a $0.1 \mathrm{~N}$ solution of $\mathrm{HNO}_{3}$. The spectrum shown in (b) was obtained for a gel prepared using nitric acid in the peptization step (at a $\mathrm{HNO}_{3}$ :ASB molar ratio $R$ of 0.14 ) and a sol aging time of $48 \mathrm{~h}$. The four $\mathrm{NO}_{3}{ }^{-}$bands marked by vertical lines are seen to be present in the gel spectrum; these nitrate-ion lines have been misinterpreted (as boehmite lines, or as lines due to organic impurities) in some previous studies of sol-gel alumina. 
lines (at 720,1049, 1348, and $1414 \mathrm{~cm}^{-1}$ ), especially the strong, sharp line at $1049 \mathrm{~cm}^{-1}$. All of these are present in both the $\mathrm{HNO}_{3}$ and the gel spectra, and all are absent in the spectrum of boehmite. These four bands correspond to $\mathrm{NO}_{3}{ }^{-}$vibrations. ${ }^{19,20}$ The symmetric-stretch nitrate line at $1049 \mathrm{~cm}^{-1}$ has been erroneously assigned to boehmite, and the asymmetric stretching line at 720 $\mathrm{cm}^{-1}$ has been erroneously assigned to boehmite gels. Also, the 1348 and $1414 \mathrm{~cm}^{-1}$ lines have been misassigned as organic features in the boehmite gels. All boehmite gel spectra that have been reported to exhibit these lines were obtained on samples that were either peptized with $\mathrm{HNO}_{3}$ or involved $\mathrm{Al}\left(\mathrm{NO}_{3}\right)_{3}$ in their synthesis. The attribution of extraneous lines to the Raman spectrum of boehmite has prevented a correct group-theoretical interpretation of the zone-center vibrations in this crystal; we plan to rectify this in a future paper.

A comparison of the spectra in the lower two panels of Fig. 5 confirms that boehmite $\mathrm{AlO}(\mathrm{OH})$ is, indeed, the primary component (other than water) in the hydrogel. The $363 \mathrm{~cm}^{-1}$ signature of the crystal is prominent in the gel, but, as we shall see, this line is actually broadened and shifted with respect to the crystal line.

\section{RAMAN SCATTERING FROM ALUMINA GELS: SYSTEMATIC CHANGES DURING SOL-GEL PROCESSING}

In the synthesis procedure for sol-gel alumina that is outlined in Fig. 1, the primary processing variable of interest in this study is the sol aging time $t_{s}$. Secondarily, we have also studied the effect of varying (or omitting) the acid peptization step. Although gel samples produced after different times $t_{s}$ all exhibit Raman spectra which, in overall appearance, resemble that shown in Fig. $5(\mathrm{~b})$, we have observed definite systematic changes as $t_{s}$ is varied. As noted in Sec. II, after gelation is achieved by concentrating and cooling the aged sol, no further structural changes are observed.

The gel Raman features that we have followed, as a function of sol aging time, are the position, linewidth, and asymmetry of the prominent boehmite band near 360 $\mathrm{cm}^{-1}$. Figure 6 shows a detailed view of this band, observed for a sample corresponding to $t_{s}=24 \mathrm{~h}$. The experimental linewidth $\Gamma_{\text {expt }}\left(\Gamma_{\text {expt }}=1 \mathrm{w}+\mathrm{rw}\right.$, where $\mathrm{lw}$ and rw are the left and right half-widths) is the observed full width at half maximum. The asymmetry is defined as $1 \mathrm{w} / \mathrm{rw}$. The experimental linewidth is a convolution of the actual linewidth $\Gamma$ and the instrumental broadening $\Gamma_{\text {inst }}, \Gamma_{\text {expt }}=\left(\Gamma^{2}+\Gamma_{\text {inst }}^{2}\right)^{1 / 2}$; in the following analysis we present the (deconvoluted) results for the estimated actual linewidth $\Gamma$.

Figure 7 shows the $360 \mathrm{~cm}^{-1}$ band for a series of samples with $t_{s}$ values varying from 1 to $72 \mathrm{~h}$. In panels (a)-(d), the experimental data are shown as solid dots, while the lines are fitted curves useful for comparing and analyzing the data. (The curves are eight-parameter fits, using two Gaussians and a linear base line. The fitted curves were used to determine the peak positions and linewidths.) Panel (e) of Fig. 7 shows a superposition of the curves; like panels (a)-(d), it demonstrates that the

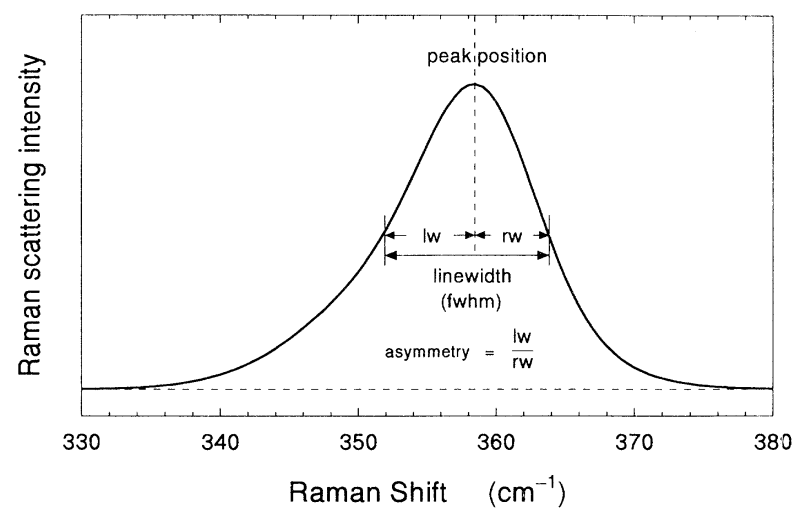

FIG. 6. Detailed view of the boehmitelike band in an alumina hydrogel, illustrating the line-shape parameters used in our analysis. The curve is a fit to data obtained for a gel synthesized using $\mathrm{HCl}$ peptization (acid:ASB molar ratio $R$ of 0.07 ) and a $t_{s}$ of $24 \mathrm{~h}$. The linewidth indicated here is the experimental linewidth; it is a convolution of the actual linewidth and the instrumental spectral slit width. The linewidth values plotted in Figs. 8 and 9 correspond to the (deconvoluted) actual linewidth.

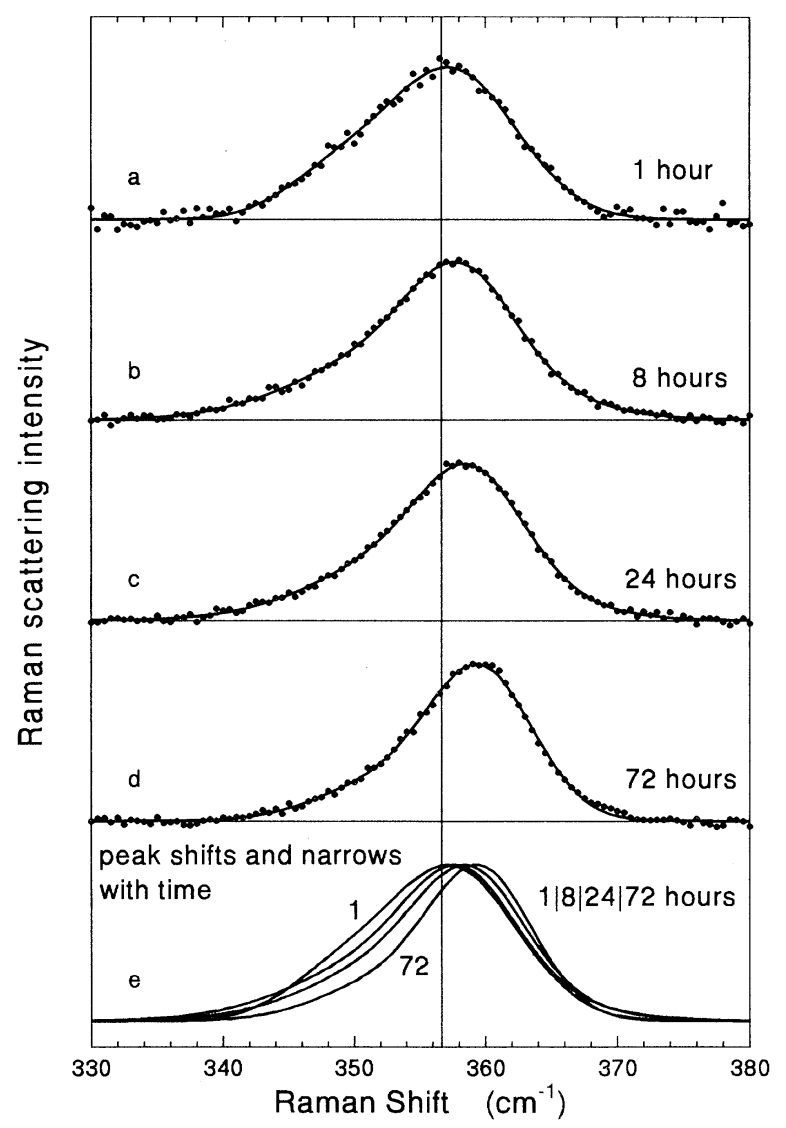

FIG. 7. The influence of sol aging time on the position and line shape of the boehmitelike band seen in the resulting hydrogels. The solid dots in panels (a)-(d) are the experimental data; the curves are fits. Panel (e) shows a superposition of the fitted curves of the top four panels, for $t_{s}$ values of $1,8,24$, and $72 \mathrm{~h}$ in order to more clearly reveal the narrowing and upshifting with increasing $t_{s}$. This series of hydrogels was prepared from a sol that had been peptized with $\mathrm{HCl}$ (with $R=0.07$ ). 
band upshifts and narrows with increasing sol aging time at $95^{\circ} \mathrm{C}$.

Figure 8, which represents our principal findings for the influence of preparation procedure on the boehmite band position and line shape in the sol-gel alumina materials, summarizes results obtained from about 70 samples, via spectra such as those in Fig. 7. Figure 8 presents the observed correlation between peak position and linewidth. The main family of materials, represented in Fig. 8 by the solid triangles, are the hydrogels prepared by the process of Fig. 1, with the peptization step carried out with nitric or hydrochloric acid (with acid:ASB ratios of 0.07 and 0.14 ). In addition, several other types of samples were investigated and have been included in Fig. 8. Boehmite itself [via more detailed data than shown in Fig. 5(c)] is included as the solid square labeled "bulk crystal" at the lower left of Fig. 8. This corresponds to the smallest linewidth and highest peak position for the $360 \mathrm{~cm}^{-1}$ band; it represents the limiting values.

The central result displayed by the extensive data contained in Fig. 8 is the position versus linewidth correlation represented by the solid straight line. This line is a fit to the gel data (the solid triangles, about 50 samples) in the upper part of the figure; the bulk-crystal point at the lower left (not included in the fit) lies close to the extrapolation of this line. Within this hydrogel data set, the gels represented by points furthest to the upper right correspond to the shortest sol aging times. With increasing gel sample $t_{s}$, the points track down and to the left, systematically heading in the direction of the bulk crystal point.

A second result is indicated by the dashed line in Fig. 8. This is a linear fit to the data set (the open squares) corresponding to the unpeptized precipitates prepared by omitting the acid addition step in Fig. 1. This family of materials also exhibits an approximately linear positionlinewidth correlation, but one that is slightly shifted with respect to the line describing the correlation for the gel samples.
Results for a few other types of sol-gel alumina samples are included in Fig. 8. The xerogels (represented by the open triangles) were made by drying selected hydrogel samples (indicated by the tie lines) at $110^{\circ} \mathrm{C}$. The $130^{\circ} \mathrm{C}$ powder precipitate was an unpeptized sample held at $130^{\circ} \mathrm{C}$ for five days in a closed vessel. For all of these samples, the position-linewidth parameters define points which lie in or near the narrow band marked by the two lines in Fig. 8. Interpretation of the correlations revealed by these data is discussed in the next section.

\section{NANOCRYSTALLINE BOEHMITE}

The points at the top right of Fig. 8, for which the boehmite-like band is substantially broadened and downshifted relative to the bulk-crystal band, correspond to alumina gels prepared using the shortest sol times $\left(t_{1}, t_{2}, \ldots\right)$ in the procedure of Fig. 1. For longer reaction times, the band exhibited by the resulting gels is seen to narrow and upshift; the spectral parameters tend toward those of the bulk crystal. This systematic variation in the Raman spectrum evidently probes a systematic variation in the structure and/or the composition of the gel, as a function of sol aging time.

The main candidate for a stoichiometric explanation of the observed Raman changes involves the possible incorporation of excess water within the boehmite phase. Intercalation of water into the interlayer (hydrogen bonding) regions in boehmite has been suggested in the literature, ${ }^{21}$ but clear evidence for this has not been presented. We consider a variation in interlayer water content to be an unlikely explanation of the results of Fig. 8. The bulk crystal is the end point corresponding to very long aging times, which seems inconsistent with an excess water explanation.

It has also been suggested that bond-length and bondangle variations might account for the increased linewidth in the gel. This idea implicitly appeals to an

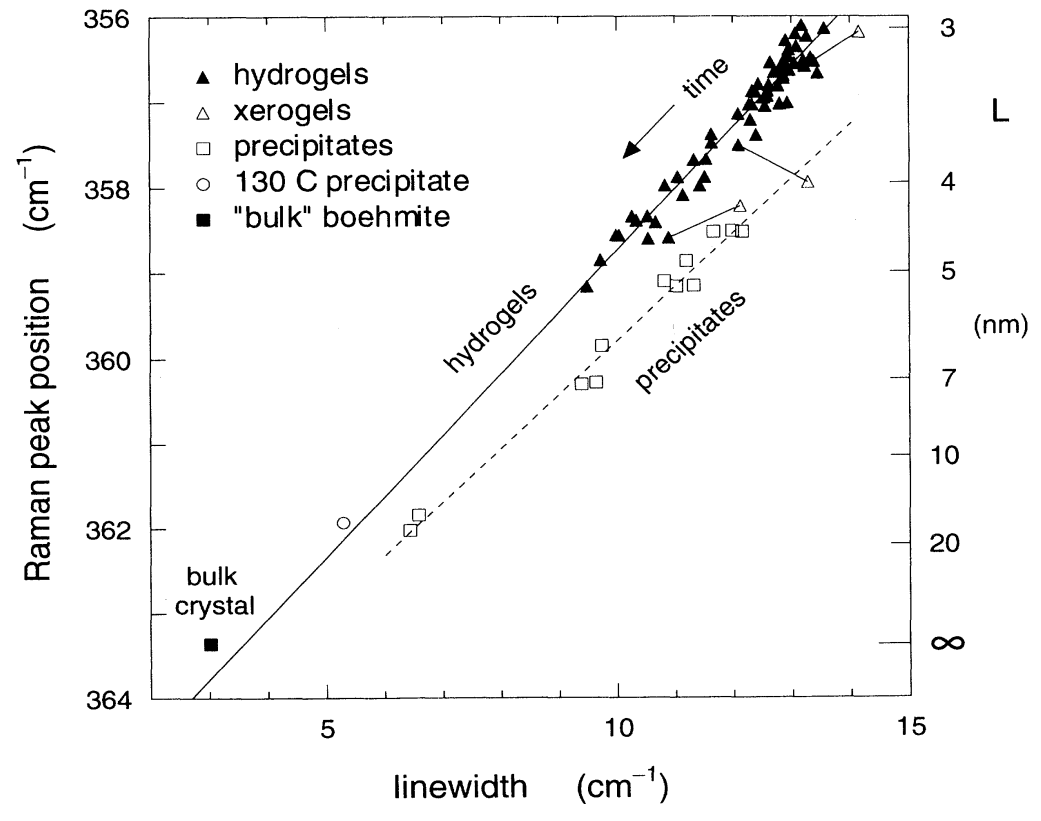

FIG. 8. The correlation between peak position and linewidth for the boehmitelike Raman band observed in sol-gel-alumina materials. Seventy samples are represented in this figure, over 50 of these being Yoldas-process hydrogels synthesized using a range of sol aging times. The other samples are described in the text. 
analogy with silica gel, which is closely related to amorphous $\mathrm{SiO}_{2}$, a covalently bonded, low coordination number, continuous random network, bulk glass that is characterized by a broad distribution of Si-O-Si bond angles. ${ }^{22}$ But the analogy between alumina gel and silica gel is wrong for many reasons. The alumina system is primarily ionic, not covalent. There is no bulk alumina glass analogous to silica glass. Coordination numbers in crystalline $\mathrm{Al}_{2} \mathrm{O}_{3}$ are significantly higher than in $\mathrm{SiO}_{2}$ : six for $\mathrm{Al}$ and four for $\mathrm{O}$ in $\mathrm{Al}_{2} \mathrm{O}_{3}$ versus four for $\mathrm{Si}$ and two for $\mathrm{O}$ in $\mathrm{SiO}_{2}$. Silica glass is a classic Zachariasen structure constructed of corner-sharing $\mathrm{SiO}_{4}$ tetrahedra; the bridging oxygens provide the flexibility needed to form the random network. $\mathrm{Al}_{2} \mathrm{O}_{3}, \mathrm{AlO}(\mathrm{OH})$, and $\mathrm{Al}(\mathrm{OH})_{3}$ are all based on edge-sharing $\mathrm{AlO}_{6}$ octahedra (nearby aluminum atoms are joined by two oxygen atoms); the geometrical constraints enforce crystalline structures. Thus, alumina gel is predominantly microcrystalline or nanocrystalline rather than amorphous, and there is little scope for bond-length or bond-angle variation.

The observed correlation between Raman linewidth and peak position, exhibited in Fig. 8 for the boehmitelike signature characteristic of the family of materials known as sol-gel alumina, bears a strong resemblance to a well-documented phenomenon observed in nanocrystalline semiconductors: the finite-size effect of nanocrystallinity (microcrystal dimensions of order $10 \mathrm{~nm}$ ) on Raman position and line shape. ${ }^{23-29}$ The gist of the mechanism is as follows. For a nanocrystal of size $L$, the strict "infinite-crystal" $k$ space selection rule is replaced by a relaxed version characterized by a $k$ space uncertainty of order $(1 / L)$. Here, $k$ is the phonon wave vector. Instead of only the $k=0$ mode of a phonon branch being Raman active (for a Raman-allowed mode symmetry), now all modes of the phonon branch within a range of $k$ values [roughly from $k=0$ up to $k=(1 / L)$ ] are Raman active. Because the phonon dispersion curve $v(k)$ is not flat, the observed Raman band shifts and broadens. The smaller is $L$, the larger is the shift and broadening of the Raman band. The $x$-ray results of the following section show, in fact, that in our materials, $L$ decreases in the decreasing time direction (the direction of increasing shift and increasing broadening) in Fig. 8. The $L$ scale along the right side of Fig. 8 is based on the $x$-ray-derived average crystallite sizes discussed in Sec. VI.

Both the clear correlation in Fig. 8 between peakposition downshift and linewidth broadening, as well as the magnitude of the measured changes in these quantities, are similar to what is observed in nanocrystalline semiconductors. A second conspicuous feature of Fig. 8 is the small but real offset of the line representing the precipitate samples relative to the line representing the main family of samples, the hydrogels. Before discussing this interesting effect, we first present, in Fig. 9, additional sets of results for the line-shape changes.

Figure 9 shows the correlation between linewidth and line-shape asymmetry (upper panel of the figure) and between peak position and asymmetry (lower panel). Here the line-shape asymmetry information is contained in the ratio $(1 \mathrm{w} / \mathrm{rw})$, where $1 \mathrm{w}$ and $\mathrm{rw}$ are the left and right half-widths illustrated in Fig. 6. For a symmetric band,

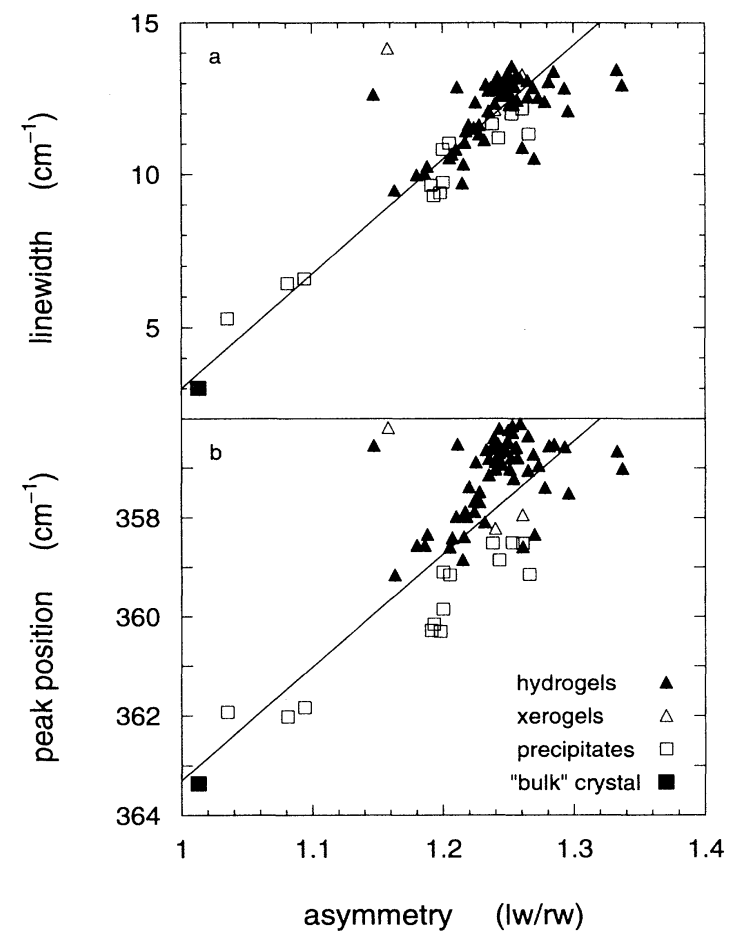

FIG. 9. Correlations between the asymmetry of the boehmitelike band line shape and the position and linewidth of the band, for the samples of Fig. 8 .

this ratio is unity; this is close to the case observed for bulk boehmite. The most asymmetric line shapes are observed for the short-time gels, for which the observed ratio is about 1.3. The asymmetry is small and thus the scatter is substantial, but Fig. 9 does show that linewidth, peak position, and asymmetry are mutually correlated.

In Fig. 8, there is an offset between the lines describing the two large populations of samples: hydrogels and precipitates. In Fig. 9(a), data from both populations overlap and no such separation or offset is evident. But in Fig. 9(b), we can again see a definite separation, as in Fig. 8. Our interpretation of these observations, in terms of nanocrystal-size arguments, is given below.

For a single nanocrystal diameter $L$, the finite-size model yields a single value for each of the three Raman band characteristics: peak position, linewidth, and lineshape asymmetry. For a distribution of nanocrystal sizes having a peak at $L$ and a spread schematically denoted by $\delta L$, the Raman peak position is determined by $L$ but the linewidth and asymmetry are increased over the single $L$ (monodisperse) value by contributions arising from the particle-size spread $\delta L$. We interpret the hydrogelprecipitate Raman band offset, discernible in Figs. 8 and 9(b) but absent in 9(a), as evidence that $\delta L$ is larger for the nanocrystal distribution in the precipitates than for the nanocrystal distribution in the hydrogels. In other words, the precipitates exhibit more inhomogeneous broadening than the hydrogels. A comparison of hydrogel and precipitate spectra, for bands with similar peak positions, reveals that the main difference is that the left half-width (lw of Fig. 6) is larger for the precipitates. 
This accounts for the offset between the two populations seen in Figs. 8 and 9(b), as well as the absence of an offset in Fig. 9(a) (since an increase in lw increases both linewidth and asymmetry, approximately preserving the linewidth-asymmetry correlation).

An increased low-frequency wing (larger lw) for the Raman band of the precipitates implies a larger spread in the particle-size distribution on the small-particle side. A possible explanation for an increase in the number of small nanocrystals is the $110^{\circ} \mathrm{C}$ drying step experienced by the precipitates (but not, of course, the hydrogels). During this step, the concentration of aluminumcontaining solute species increases drastically, and at some point it exceeds the critical supersaturation for nucleation. The resulting additional burst of nucleation gives rise to a population of small nanocrystals, which is responsible for the increased dispersion on the small- $L$ side of the particle-size distribution.

To test this idea, we subjected three of the hydrogels to the same $110^{\circ} \mathrm{C}$ drying step experienced by the precipitates. The results are included in Fig. 8 as the three pairs of triangles connected by tie lines. For each pair of points, the filled triangle corresponds to the initial hydrogel and the open triangle corresponds to the xerogel produced by drying that hydrogel at $110^{\circ} \mathrm{C}$. All three tie lines move the initial point to the right, to larger linewidth. Two of the xerogel points are seen to lie near the line describing the precipitates, while the smallest- $L$ case has moved part way in the same general direction. These results are consistent with the picture of increased small-particle dispersion caused by a late, drying-induced burst of nucleation.

The open circle in Fig. 8 is for a precipitate which, directly after formation by hydrolysis and condensation in the 1:110 ASB/water liquid, was transferred (precipitate plus liquid) to a closed pressure vessel and held at $130^{\circ} \mathrm{C}$ for five days. It was then removed and dried at $110^{\circ} \mathrm{C}$, leaving a white powder. This sample can be viewed as an exercise in boehmite crystal growth. In Fig. 8 , its position is the closest one to the bulk-crystal point. It is also worth noting that it lies near the main line rather than the offset line, showing that the offset is not associated with the absence of acid (since this sample was acid-free).

The nanocrystal particles in contact with the sol (the nanocrystals in the colloidal sol, for the liquids that precede the hydrogels, and the nanocrystals forming the precipitate for the unpeptized stirred suspensions) are presumed to grow in size by the dissolution and reprecipitation of an amorphous Al-containing component, or possibly an assembly of clusters, that is formed in the initial hydrolysis and condensation reactions between ASB and water. Such a source of alumina can account for the growth of the boehmite signal that we see. In the case of the $130^{\circ} \mathrm{C}$ precipitate discussed above, it appears that the source has been depleted during the five day period of crystal growth, since we see no spectral evidence of a final burst of nucleation caused by the drying step.

The crystalline character of all of these sol-gel materials (hydrogels, xerogels, precipitates) is attested to by the unambiguous signature of crystalline boehmite in the Ra- man spectra. The six strongest lines of the crystal (all those listed as medium or stronger in Table I, except for the $733 \mathrm{~cm}^{-1}$ line) all appear in the gel spectra. In addition, transmission electron diffraction on gel films (prepared by allowing an aged sol to dry by evaporation on a sample grid) exhibited many sharp diffraction rings. $X$-ray-diffraction data, described in the next section, confirm the crystallinity and provide a valuable calibration of microcrystallite size.

\section{X-RAY-DIFFRACTION DETERMINATION OF NANOCRYSTAL SIZES}

X-ray-diffraction experiments were performed on several of the materials for which Raman results were given in Fig. 8. Measurements were done on samples in powder form, as described in Sec. II D. The x-ray linewidth results were used to estimate average crystallite sizes by means of the Scherrer equation, Eq. (1).

Figure 10 shows the $x$-ray diffraction data. The peak

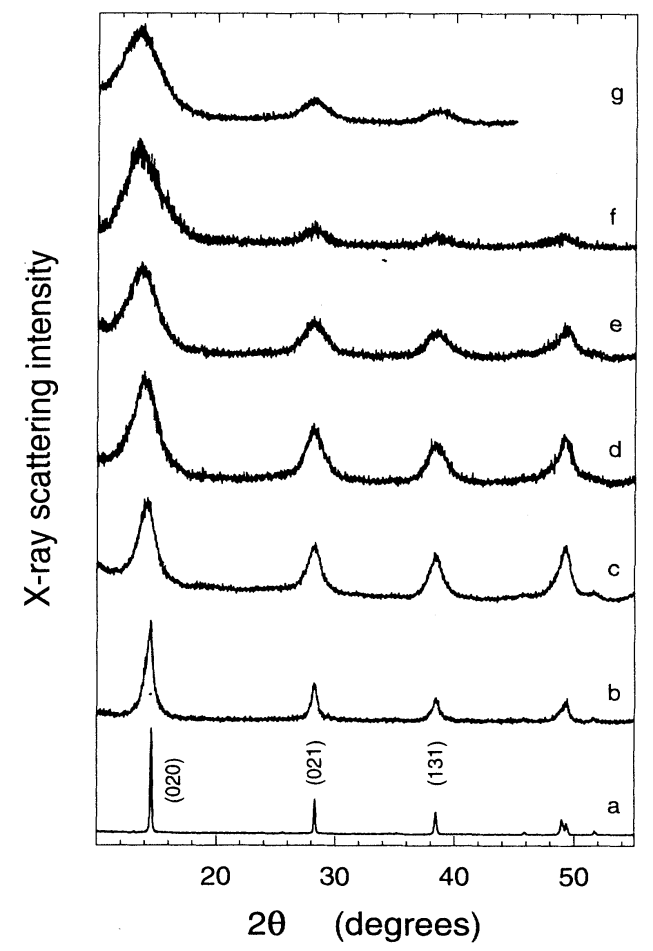

FIG. 10. X-ray-diffraction data taken on powder samples, using $\mathrm{Cu} K \alpha$ radiation (1.54 $\AA$ wavelength). The very sharp peaks in trace a, obtained with micron-size crystals, are consistent with the calculated diffraction spectrum of bulk crystalline boehmite. The peaks in traces b-g show progressively broader linewidths, indicating progressively smaller crystallite sizes. Crystallite sizes were calculated, using the Scherrer formula [Eq. (1)], from the widths of the $(021)$ and (131) peaks. The bulk-crystal sample corresponding to trace $\mathrm{a}$ is represented by a solid square in the Raman data of Figs 8,9 , and 11 . Trace $b$ is the $130^{\circ} \mathrm{C}$ precipitate described in the text and is represented by an open circle in Figs. 8 and 11 . Traces c-e are 48, 8, and $1 \mathrm{~h}$ precipitates, respectively (open squares in Figs. 8, 9, and 11). Trace $\mathrm{f}$ is for a $48 \mathrm{~h}$ gel peptized with nitric acid at $R=0.07$ (solid triangle in Figs. 8, 9, and 11). Trace $\mathrm{g}$ is for a $1 \mathrm{~h}$ xerogel (open triangle in the upper right-hand corner of Figs. 8 and 11). 
assignments are given with respect to the $\gamma-\mathrm{AlO}(\mathrm{OH})$ orthorhombic $\mathrm{Cmcm}$ crystal structure. ${ }^{11,30}$ At the bottom (trace a) is the result obtained for a powder composed of micron-size crystals; this trace exhibits very narrow linewidths (about $0.1^{\circ}$ ) and is the pattern expected for bulk crystalline boehmite. The traces $b-g$ show progressively broader linewidths (up to a few degrees), indicating progressively smaller crystallite sizes. The low-angle (020) band becomes asymmetric and shifts to higher d values as the linewidths increase, while the (021) and (131) bands remain symmetric and do not shift with linewidth broadening. The (021) and (131) peaks were used for crystallite size determination by the Scherrer method. They gave consistently similar results, as seen in Fig. 11.

Figure 11 presents the correlation between the crystallite size, as determined by x-ray diffraction, and the Raman peak position and linewidth (of the $360 \mathrm{~cm}^{-1}$ band) observed for the same sample. The symbols used in this figure correspond to the same convention used in Fig. 8. For each Raman determination (vertical location) there are two x-ray determinations (horizontal locations), corresponding to the (021) and (131) diffraction peaks. The range of crystallite sizes is seen to extend down to about 3 $\mathrm{nm}$ (justifying our use of the terms nanocrystal and nanocrystallinity).

The top $x$-axis scale in Fig. 11 gives the crystallite size $L$; the bottom $x$-axis scale gives $L^{-1}$. The scale along the upper right side gives the downshift $\Delta \bar{v}$ of the Raman

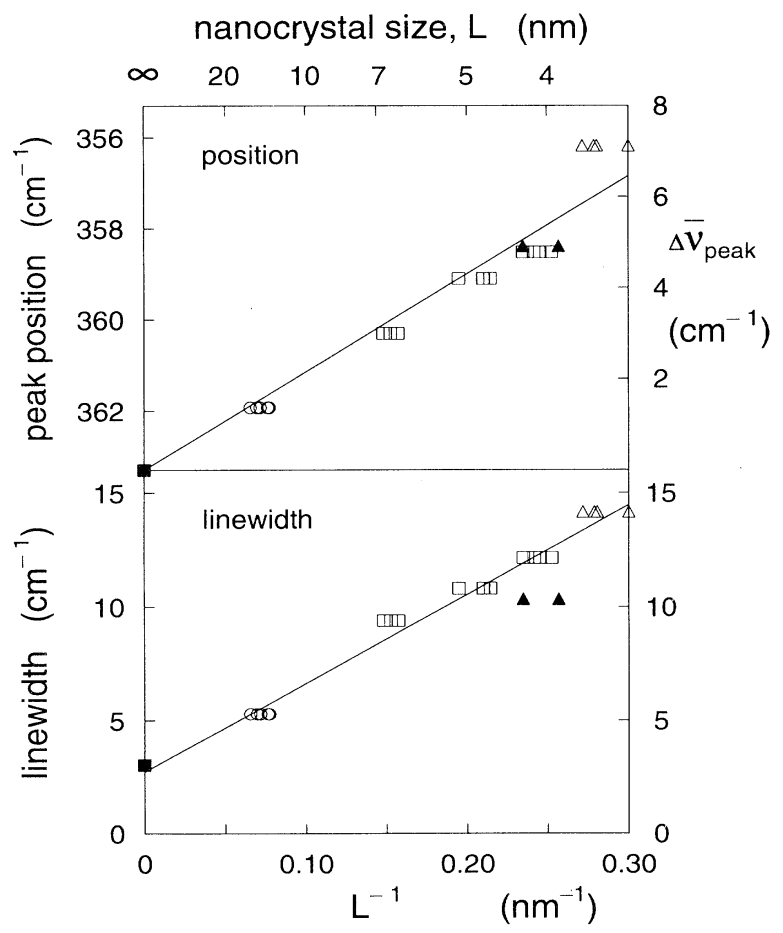

FIG. 11. The correlation between the crystallite size $(L)$, as determined by $x$-ray diffraction for a particular sample, and the Raman peak position and linewidth (of the $360 \mathrm{~cm}^{-1}$ band) observed for the same sample. The samples and their corresponding symbols have been described in the caption of Fig. 10. peak position, relative to the peak position for the infinite crystal. The two straight lines are least-squares linear-in$L^{-1}$ fits to the data:

$$
\Delta \bar{v}=k_{1}(1 / L)
$$

and

$$
\Gamma=k_{2}(1 / L)+\Gamma_{0},
$$

where $\Gamma$ is the linewidth, and $k_{1}, k_{2}$, and $\Gamma_{0}(21.5$ $\mathrm{cm}^{-1} \mathrm{~nm}, 39.2 \mathrm{~cm}^{-1} \mathrm{~nm}$, and $2.7 \mathrm{~cm}^{-1}$, respectively) are determined from the fit. These calibration relations, connecting Raman peak-position and linewidth with crystallite size, were used to obtain the right-hand scale as in Fig. 8. They are also used in the next section, to determine $L\left(t_{s}\right)$ growth kinetics.

A linear relationship between Raman peak position and $L^{-\alpha}$, with $\alpha=1.0$, has also been seen in graphite ${ }^{28}$ and boron nitride. ${ }^{29}$ It is noteworthy that boehmite, like graphite and BN, possesses a layered structure (as discussed in Sec. III). In terms of $\alpha$, which is an empirical Raman-versus-size scaling exponent, these layered materials behave differently than the covalent network semiconductors silicon ${ }^{31,32}$ and gallium arsenide. ${ }^{23,33}$ Our analysis of the reported Raman results ${ }^{23,31-33}$ for the network semiconductors yields, for those materials, a scaling exponent $\alpha$ of about $1.5 .^{34}$

\section{KINETICS OF NANOCRYSTAL GROWTH}

Figure 12 displays the results of individual samplepreparation runs, showing the dependence of the boehmite signature Raman band peak position of the hydrogel on the sol aging time ( $t_{s}$ in Fig. 1). Results are shown for

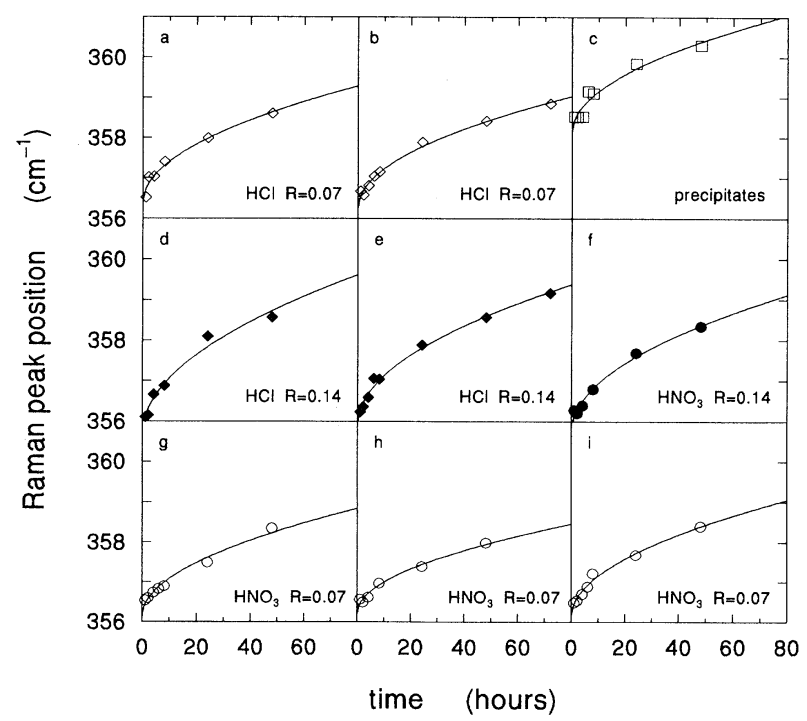

FIG. 12. The results of individual runs on the influence of the sol aging time on the Raman peak position of the boehmitelike band in the hydrogel. The acid used in the sol peptization step is indicated in each case, as well as the acid:ASB molar ratio $R$. One unpeptized example $(R=0)$ is included in panel $\mathrm{c}$ for comparison. The peak-position scale in this figure is reversed with respect to the scales used in Figs. 8 and 9(b). 
both of the acids used in the peptization step, $\mathrm{HCl}$ and $\mathrm{HNO}_{3}$, and for both acid concentrations used, $R=0.07$ and $R=0.14$ ( $R$ is $\mathrm{H}: \mathrm{Al}$, the acid:ASB mole ratio). Also included (panel c) are results for the precipitates obtained in an acid-free run. All of the runs were well represented by fits of the form

$$
\bar{v}(t)-\bar{v}(0)=r t^{1 / 2} \text {. }
$$

The curves shown in Fig. 12 correspond to Eq. (4), with the best-fit parameters, $\bar{v}(0)$ and $r$, listed in Table II. The precipitate behavior clearly differs from the rest in its larger $\bar{v}(0)$. For the gels, $\bar{v}(0)$ is smaller for the higher acid concentration, but there is no discernible difference between the use of $\mathrm{HCl}$ and $\mathrm{HNO}_{3}$ as peptizing agent. The rate constant $r$ increases with increasing acid concentration.

As the characteristic microcrystallite size increases, the finite-size-induced downshift of the Raman peak position is reduced, and the peak moves upward toward its bulk-crystal value of $363 \mathrm{~cm}^{-1}$. Using the results of the last section, we can make this interpretation quantitative by invoking the $\Delta \bar{v}(L)$ scaling relation of Eq. (2), which connects the left and right scales of Fig. 8. Each set of $\bar{v}(t)$ data, corresponding to the set of points contained in a panel of Fig. 12, is thus transformed to a set of $L(t)$ data, with $L$ the crystallite size. The results obtained for panels $\mathrm{c}$ and e of Fig. 12 are presented in Fig. 13.

All of the hydrogels represented in Fig. 12 yield $L(t)$ results similar to the $\mathrm{HCl} R=0.14$ data (panel e of Fig. 12, lower part of Fig. 13) shown in the lower part of Fig. 13. The precipitate run (panel c of Fig. 12, upper part of Fig. 13) is distinctly different. The curves shown in Fig. 13 are fits of the form,

$$
\left[L_{3}(t)\right]^{3}-\left[L_{e}(0)\right]^{3}=S_{3} t,
$$

where $L_{3}(0)$ and $S_{3}$ are the fit parameters. This form was found to adequately describe all of the $L(t)$ results; the values obtained for $L_{3}(0)$ and $S_{3}$ are given in the last two columns of Table II. Also included in Table II are results to fits of the form,

$$
\left[L_{2}(t)\right]^{2}-\left[L_{2}(0)\right]^{2}=S_{2}(t)
$$

that also describes the experimental results, within the statistical error bars shown.

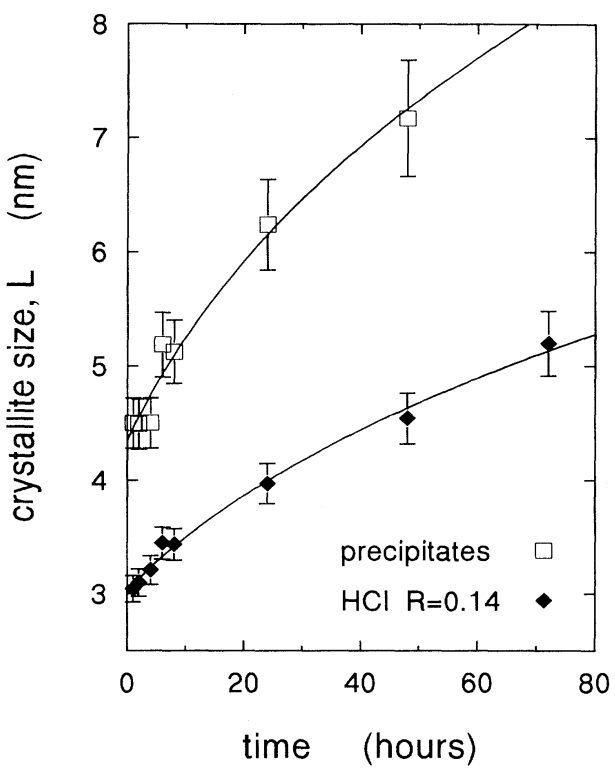

FIG. 13. Nanocrystal-size growth kinetics for the runs of Figs. 12(c) and 12(e), estimated from the Raman and x-ray data by the method described in the text.

The time dependence exhibited by $L_{2,3}(t)$ is similar to that found in various theories of crystal growth, ${ }^{35,36}$ which come under the general heading of Ostwald ripening. Ostwald ripening (coarsening, coalescence, or just ripening) is basically a zero-sum game in which larger particles grow at the expense of smaller particles; for our purposes, the larger particles are nanocrystalline boehmite, and the smaller particles are amorphous or incompletely crystallized $\mathrm{AlO}(\mathrm{OH})$. Our results are quite consistent with the often invoked cubic-law behavior [Eq. (5)] corresponding to the fitted curves in Fig. 13, but our data are not accurate enough to rule out parabolic-law behavior [Eq. (6)].

The unpeptized precipitate sample included in Figs. 12 and 13 and Table II is characterized by a larger initial crystallite size $[L(0)]$ and by a faster growth rate $(s)$ than the peptized sols. Acid peptization limits early crystal growth. Overall, both acids used for preparation of the sols yield quite similar results. No difference is ob-

TABLE II. Parameters obtained from the rate-equation fits [Eqs. (4)-(6) in the text] to the data of Figs. 12 and 13.

\begin{tabular}{lcccccccc}
\hline $\begin{array}{c}\text { Panel } \\
\text { in Fig. 12 }\end{array}$ & Acid & $\begin{array}{c}\text { Acid concentration } \\
(\mathrm{H}: \mathrm{Al})\end{array}$ & $\begin{array}{c}v(0) \\
\left(\mathrm{cm}^{-1}\right)\end{array}$ & $\begin{array}{c}r \\
\left(\mathrm{~cm}^{-1} \mathrm{~h}^{-1 / 2}\right)\end{array}$ & $\begin{array}{c}L_{2}(0) \\
(\mathrm{nm})\end{array}$ & $\begin{array}{c}s_{2} \\
\left(\mathrm{~nm}^{2} \mathrm{~h}^{-1}\right)\end{array}$ & $\begin{array}{c}L_{3}(0) \\
(\mathrm{nm})\end{array}$ & $\begin{array}{c}s_{3} \\
\left(\mathrm{~nm}^{3} \mathrm{~h}^{-1}\right)\end{array}$ \\
\hline $\mathrm{a}$ & $\mathrm{HCl}$ & $R=0.07$ & 356.4 & 0.32 & 3.3 & 0.22 & 3.3 & 1.3 \\
b & $\mathrm{HCl}$ & $R=0.07$ & 356.3 & 0.31 & 3.2 & 0.19 & 3.2 & 1.1 \\
$\mathrm{c}$ & none & $R=0.0$ & 358.1 & 0.33 & 4.5 & 0.70 & $4.4^{\mathrm{a}}$ & $6.3^{\mathrm{a}}$ \\
$\mathrm{d}$ & $\mathrm{HCl}$ & $R=0.14$ & 355.7 & 0.44 & 3.0 & 0.27 & 3.0 & 1.5 \\
$\mathrm{e}$ & $\mathrm{HCl}$ & $R=0.14$ & 355.9 & 0.39 & 3.1 & 0.24 & $3.0^{\mathrm{a}}$ & $1.5^{\mathrm{a}}$ \\
$\mathrm{f}$ & $\mathrm{HNO}_{3}$ & $R=0.14$ & 355.8 & 0.38 & 3.0 & 0.21 & 3.0 & 1.2 \\
$\mathrm{~g}$ & $\mathrm{HNO}_{3}$ & $R=0.07$ & 356.1 & 0.30 & 3.1 & 0.18 & 3.1 & 1.0 \\
$\mathrm{~h}$ & $\mathrm{HNO}_{3}$ & $R=0.07$ & 356.2 & 0.25 & 3.2 & 0.14 & 3.1 & 0.7 \\
i & $\mathrm{HNO}_{3}$ & $R=0.07$ & 356.1 & 0.33 & 3.2 & 0.20 & 3.1 & 1.1 \\
\hline \hline
\end{tabular}

${ }^{\text {a }}$ The curves that correspond to these parameters are shown in Fig. 13. 
served between $\mathrm{HCl}$ and $\mathrm{HNO}_{3}$ in terms of the $L(0)$ values obtained, but increasing the the acid concentration from $R=0.07$ to $R=0.14$ does reduce $L(0)$ slightly, an effect which may reflect faster peptization (and reduced opportunity for early growth) at the higher concentration. The subsequent growth rate seems to be slightly larger with $\mathrm{HCl}$ than with $\mathrm{HNO}_{3}$ and, for both acids, growth occurs somewhat more quickly at the higher acid concentration. But in all cases the growth is very slow, so that the gelation step yields hydrogels of nanocrystalline boehmite.

\section{SUMMARY}

A systematic Raman-scattering investigation has been carried out on sol-gel alumina prepared by the Yoldas process of Fig. 1, primarily as a function of the sol aging time, but also, secondarily, as a function of the acid peptization step (including its omission). The predominant aluminum-containing component of the alumina hydrogels and precipitates is nanocrystalline boehmite, $\gamma-\mathrm{AlO}(\mathrm{OH})$, and in the course of this work we have clarified certain aspects of the boehmite Raman spectrum.

A careful study was made of small but systematic changes observed, as a function of the process parameters, in the line shape and position of the dominant boehmite band near $360 \mathrm{~cm}^{-1}$. The position-linewidth correlation established by the data in Fig. 8, as well as the more subtle line-shape asymmetry correlations of Fig. 9, have been interpreted in terms of nanocrystallinityinduced finite-size effects associated with the slow growth of the $\gamma-\mathrm{AlO}(\mathrm{OH})$ nanocrystals during the sol phase. $\mathrm{X}$-ray-diffraction experiments were used to determine nanocrystal sizes (as small as $3 \mathrm{~nm}$ for gels prepared from fresh sols) and to estimate growth kinetics from the $\mathrm{Ra}$ man line-shape results. The Raman peak-position shift is proportional to $L^{-\alpha}$, where $L$ is the average nanocrystal size and $\alpha$ is a Raman-versus-size scaling exponent. For $\mathrm{AlO}(\mathrm{OH})$ we find $\alpha$ to be 1.0 , close to scaling-exponent values reported for graphite and $\mathrm{BN}$ and different from the value of 1.5 which describes the reported behavior of $\mathrm{Si}$ and GaAs.

\section{ACKNOWLEDGMENTS}

This research was supported in part by Philip Morris, U.S.A. We are grateful to A. G. Kallianos and A. C. Lilly for their advice and encouragement in the initial phase of this work. We are indebted to $\mathrm{K}$. Wefers for providing the crystalline boehmite sample and G. V. Gibbs for making available to us the Bukowinski-Downs crystalstructure graphics program used in constructing Fig. 2. S. Joardar provided invaluable assistance in materials synthesis techniques. We thank K. Wefers, N. Rivier, and A. L. Ritter for valuable discussions, S. McCartney for electron diffraction and TEM work, and T. N. Solberg and $M$. Ferguson for very valuable help with the $\mathrm{x}$-raydiffraction experiments.
${ }^{1}$ B. E. Yoldas, Am. Ceram. Soc. Bull. 54, 286 (1975).

${ }^{2}$ B. E. Yoldas, Am. Ceram. Soc. Bull. 54, 289 (1975).

${ }^{3}$ B. E. Yoldas, J. Mater. Sci. 10, 1856 (1975).

${ }^{4}$ B. E. Yoldas, J. Appl. Chem. Biotechnol. 23, 803 (1973).

${ }^{5}$ C. J. Brinker and G. W. Scherer, Sol-Gel Science (Academic, Boston, 1990), p. 599.

${ }^{6}$ Temperature is an important variable in the Yoldas process. If cool water is used, $\mathrm{Al}(\mathrm{OH})_{3}$ predominates and forms mixedphase precipitates. A Raman-scattering investigation of the distinction between the "high-temperature" sol-gel regime for this system (temperatures close to $95^{\circ} \mathrm{C}$ ) and the "lowtemperature" precipitate regime (temperatures close to $25^{\circ} \mathrm{C}$ ) will be presented by us in a subsequent paper. The present paper is concerned with the high-temperature regime that is optimum for the preparation of alumina gels.

${ }^{7}$ C. J. Brinker and G. W. Scherer, Sol-Gel Science (Academic, Boston, 1990), p. 371.

${ }^{8}$ Our simple deconvolution is a standard approximation which is strictly correct for Gaussians. A more sophisticated analysis for Lorentzians has been given by J. GonzalezHernandez, G. H. Azarbayejani, R. Tsu, and F. H. Pollak, Appl. Phys. Lett. 47, 1350 (1985). Since, for most of our samples, $\Gamma_{\text {expt }}$ is substantially greater than $\Gamma_{\text {inst }}$, the instrumental correction is small and $\Gamma$ is insensitive to the deconvolution procedure.

${ }^{9}$ A. C. Pierre and D. R. Uhlmann, in Better Ceramics Through Chemistry, edited by C. J. Brinker, D. E. Clark, and D. R. Ulrich (Elsevier, New York, 1984), p. 119.

${ }^{10}$ B. E. Warren, $X$-ray Diffraction (Addison-Wesley, Reading, MA, 1969), pp. 253 and 258; H. P. Klug and L. E. Alexander,
$X$-ray Diffraction Procedures for Polycrystalline and Amorphous Materials (Wiley, New York, 1973), pp. 687 and 635.

${ }^{11}$ C. E. Corbato, R. T. Tettenhorst, and G. G. Christoph, Clays Clay Miner. 33, 71 (1985).

${ }^{12}$ Figure 2 was constructed using the atom positions of Corbato, Tettenhorst, and Christoph (Ref. 11), with the aid of a crystal-structure graphics program written by $\mathrm{M}$. Bukowinski and R. Downs.

${ }^{13}$ S. P. S. Porto and R. S. Krishnan, J. Chem. Phys. 47, 1009 (1967).

${ }^{14}$ A. S. Barker, Phys. Rev. 132, 1474 (1963).

${ }^{15}$ This sample, prepared at Alcoa Laboratories, was made by autoclaving aluminum metal in excess water. X-ray diffraction, performed at NASA Langley, confirmed that the material was well-crystallized boehmite.

${ }^{16}$ A. B. Kiss, G. Keresztury, and L. Farkas, Spectrochim. Acta, Part A 36, 653 (1980). Peak positions reported in this paper agree well with our results of Table I, with one notable exception (in addition to the $\mathrm{NO}_{3}{ }^{-}$modes discussed in the text). Kiss, Keresztury, and Farkas place the dominant line at 369 $\mathrm{cm}^{-1}$ instead of $363 \mathrm{~cm}^{-1}$. Since our result was repeatedly checked and is also consistent with the asymptotic limit of the family of gel samples, the $363 \mathrm{~cm}^{-1}$ value is the correct one.

${ }^{17}$ G. Mariotto, E. Cazzanelli, G. Carturan, R. Di Maggio, and P. Scardi, J. Solid State Chem. 86, 263 (1990).

${ }^{18}$ T. Assih, A. Ayral, M. Abenoza, and J. Phalippou, J. Mater. Sci. 23, 3326 (1988).

${ }^{19}$ D. E. Irish and A. R. Davis, Can. J. Chem. 46, 943 (1968); D. E. Irish and G. E. Walrafen, J. Chem. Phys. 46, 378 (1967); 
D. E. Irish, in Ionic Interactions from Dilute Solutions to Fused Salts, edited by S. Petrucci (Academic, New York, 1971), p. 187.

${ }^{20}$ P. Scardi, J. Solid State Chem. 86, 263 (1990).

${ }^{21} \mathrm{~K}$. Wefers and C. Misra, Oxides and Hydroxides of Aluminum, ALCOA (Alum. Co. Am.) Res. Lab, Tech. Pap 19, revised (ALCOA Laboratories, ALCOA Center, 1987).

${ }^{22}$ R. Zallen, The Physics of Amorphous Solids (Wiley, New York, 1983).

${ }^{23}$ K. K. Tiong, P. M. Amirtharaj, F. H. Pollak, and D. E. Aspnes, Appl. Phys. Lett. 44, 122 (1984); M. Holtz, R. Zallen, O. Brafman, and S. Matteson, Phys. Rev. B 37, 4609 (1988).

${ }^{24}$ I. H. Campbell and P. M. Fauchet, Solid State Commun. 58, 739 (1986).

${ }^{25}$ R. Zallen, J. Non-Cryst. Solids 141, 227 (1992).

${ }^{26}$ T. Kanata, H. Murai, and K. Kubota, J. Appl. Phys. 61, 969 (1987).

${ }^{27}$ S. J. Yu, H. Asahi, S. Emura, H. Sumida, S. Gonda, and J. Tanoue, J. Appl. Phys. 66, 856 (1989).

${ }^{28}$ K. Nakamura, M. Fujitsuka, and M. Kitajima, Phys. Rev. B 41, 12260 (1990).

${ }^{29}$ R. J. Nemanich, S. A. Solin, and R. M. Martin, Phys. Rev. B 23, 6348 (1981).
${ }^{30}$ G. G. Christoph, C. E. Corbato, D. A. Hofmann, and R. T. Tettenhorst, Clays Clay Miner. 27, 81 (1979).

${ }^{31}$ H. Richter, Z. P. Wang, and L. Ley, Solid State Commun. 39, 625 (1981).

${ }^{32}$ Z. Iqbal and S. Veprek, J. Phys. C 15, 377 (1982).

${ }^{33}$ M. Gargouri, B. Prevot, and C. Schwab, J. Appl. Phys. 62, 3902 (1987).

${ }^{34}$ It is provocative that, for the cases examined, $\alpha$ is about equal to $d / 2$, where $d$ is the network dimensionality (e.g., two for graphite, three for silicon); see R. Zallen, The Physics of Amorphous Solids (Wiley, New York, 1983), p. 89.

${ }^{35}$ I. M. Lifshitz and V. V. Slyozov, J. Phys. Chem. Solids 19, 35 (1961); C. Wagner, Z. Elektrochem. 65, 581 (1961); W. W. Mullins, J. Appl. Phys. 59, 1341 (1986); N. Rivier, in Finite Systems: From Clusters to Crystals, edited by P. Jena, S. N. Khanna, and B. K. Rao (Kluwer, Dordrecht, 1992), p. 189; P. W. Voorhees and M. E. Glicksman, Metall. Trans. 15A, 1081 (1984).

${ }^{36} \mathrm{~A}$ simple argument for the parabolic law is available for growth that is driven by crystallite curvature; growth rate $(d / d t) L^{3}$ is proportional to surface area $\left(L^{2}\right)$ and to curvature $(1 / L)$, so that $L(d L / d t)$ is constant and $L^{2}$ increases linearly with time. 